\title{
Responsabilidad Civil del Cirujano-Dentista: Análisis de las Demandas Presentadas en el Municipio de Ribeirão Preto-São Paulo, Brasil
}

\author{
Civil Liability of Dental Surgeon: Legal Proceedings Analysis \\ in the City of Ribeirao Preto, São Paulo State, Brazil
}

\begin{abstract}
Andrea Sayuri Silveira Dias Terada*; Laís Gomes de Araujo*; Marta Regina Pinheiro Flores $^{* *} \&$ Ricardo Henrique Alves da Silva ${ }^{* * *}$
\end{abstract}

TERADA, A. S. S. D.; DE ARAUJO, L. G.; FLORES, M. R. P. \& DA SILVA, R. H. A. Responsabilidad civil del cirujano-dentista: Análisis de las demandas presentadas en el municipio de Ribeirão Preto-SP, Brasil. Int. J. Odontostomat., 8(3):365-369, 2014.

RESUMEN: El objetivo del trabajo fue realizar una revisión de los procesos judiciales que involucran demandas de responsabilidad civil interpuestas contra cirujanos-dentistas en el Forum de Ribeirão Preto, Estado de São Paulo, Brasil. Fue realizada una búsqueda nominalde profesionales y clínicas, debidamente inscritos en el Consejo Regional de Odontología de São Paulo, involucrados en procesos civiles en el municipio de Ribeirão Preto - São Paulo. Como resultado fue observado un significativo aumento $(73,3 \%)$ en el número de demandas presentadas en los últimos cinco años, siendo la especialidad de prótesis dental $(35,6 \%)$ la más involucrada. Se puede concluir que es importante el conocimiento por parte del profesional en cuanto al aumento de divergencias en la relación profesional/paciente y algunas medidas son necesariaspara su prevención, para que los cirujanos-dentistas busquen una práctica profesional más segura.

PALABRAS CLAVE: cirujano-dentista, odontología legal, responsabilidad civil, procesos.

\section{INTRODUCCIÓN}

Con el objetivo de sustentar el equilibrio en la sociedad, son establecidos derechos y deberes por medio de normativas jurídicas, las cuales permiten limitar las relaciones humanas (Nader, 2003). Los cirujanos-dentistas, además de responder como ciudadanos, responden por las acciones ejercidas en su actividad profesional estando sujetos a las obligaciones de orden penal, civil, ético y administrativo (Simonetti, 1999; Serra, 2001).

Actualmente, se observan muchos casos de insatisfacción relacionados con el tratamiento odontológico recibido, hecho que, consecuentemente, genera un aumento del número de demandas presentadas contra cirujanos-dentistas (de Paula et al., 2010). Los pacientes pueden asimismo ser incentivados por sus círculos sociales o por los propios medios a buscar resarcimientos pecuniarios con- tra sus dentistas (Kfouri-Neto, 2002; Silva, 2010; Santos et al., 2010). La divulgación y el amplio acceso a las normas del derecho brasileño pueden explicar este creciente número (Senado do República Federativa do Brasil, 1988; 1990; 2002). Tal hecho, generó un análisis mayor de la calidad del servicio odontológico prestado (Ribeiro, 1996; Tanaka, 2002).

En este contexto, cobra importancia el instituto de la responsabilidad civil, que se caracteriza como una de las formas más eficaces de restituir el daño sufrido, garantizando a la víctima el resarcimiento del perjuicio por el agente que causó el daño (Pereira,1998). El presente estudio tiene como objetivo evaluar los procesos de responsabilidad civil instaurados contra cirujanos-dentistas y clínicas odontológicas, en el municipio de Ribeirão Preto, Estado de São Paulo, Brasil.

Cursando doctorado en Ciencias, Facultad de Medicina de Ribeirão Preto, Universidad de São Paulo, Ribeirão Preto, Brasil.

" Cursando maestría en Biología Oral, Facultad de Odontología de Ribeirão Preto, Universidad de São Paulo, Ribeirão Preto, Brasil.

*** Profesor del Departamento de Estomatología, Salud Pública y Odontología Forense, Odontología Forense, Facultad de Odontología de Ribeirão Preto, Universidad de São Paulo, Ribeirão Preto, Brasil. 


\section{MATERIAL Y MÉTODO}

Una lista con los nombres de los cirujanos-dentistas y clínicas inscritos en el municipio de Ribeirão Preto (municipio situado en la parte noroeste del estado de São Paulo, con una población estimada de poco más de 600.000 habitantes) fue solicitada al Consejo Regional de Odontología. Fueron constatados 1930 profesionales y 264 clínicas registradas. A partir de estos nombres fue realizada una búsqueda nominal on line, analizando la participación como parte demandada en algún proceso por medio del banco de datos del Tribunal de Justicia de São Paulo.

En esta búsqueda fue posible observar 145 procesos civiles involucrando a profesionales de Odontología y establecimientos Odontológicos planteados. Posteriormente, un análisis directo fue realizado en las 10 cámaras civiles del Foro de Ribeirão Preto São Paulo, para verificar si estas controversias involucraban la actividad profesional, y si éstas estaban relacionadas con reclamos por parte de los pacientes, en relación al tratamiento odontológico recibido. En los procesos encontrados fueron verificados los siguientes elementos: el año en que la demanda fue interpuesta; la especialidad profesional involucrada; la indemnización solicitada; la existencia de seguro de responsabilidad civil por parte del cirujano-dentista; la presencia del laudo pericial y; la conclusión del proceso (si fuese pertinente).
Una adaptación del Coeficiente de Experiencia Procesal (CEP) propuesto por de Paula et al., que relaciona la cantidad de demandas presentadas con el número de cirujanos-dentistas, multiplicado por la base 1000 (mil), fue utilizada en este trabajo para posibilitar un análisis de la importancia que este número de procesos representa para el municipio en el año 2011. En este estudio fueron incluidos procesos involucrando clínicas odontológicas ya que requiere un récord para la profesión en Brasil, que se puede hacer en nombre del profesional o clínica. Por esta razón, el CEP se adaptó y por este motivo el número de clínicas registradas en el consejo fue sumado al número de cirujanos-dentistas, conforme a Figura 1:

Coeficiente de Experiencia Procesal

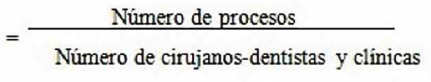

$\mathrm{X} 1000$

Fig. 1. Coeficiente de Experiencia Procesal.

\section{RESULTADOS}

En la etapa de los procesos de esta investigación, de los 145 procesos encontrados en el banco de datos judiciales involucrando a los cirujanos-dentistas y clínicas odontológicas, 45 de ellos acusaban daño por parte de los pacientes en relación al tratamiento odontológico recibido. En cuanto al número de deman-

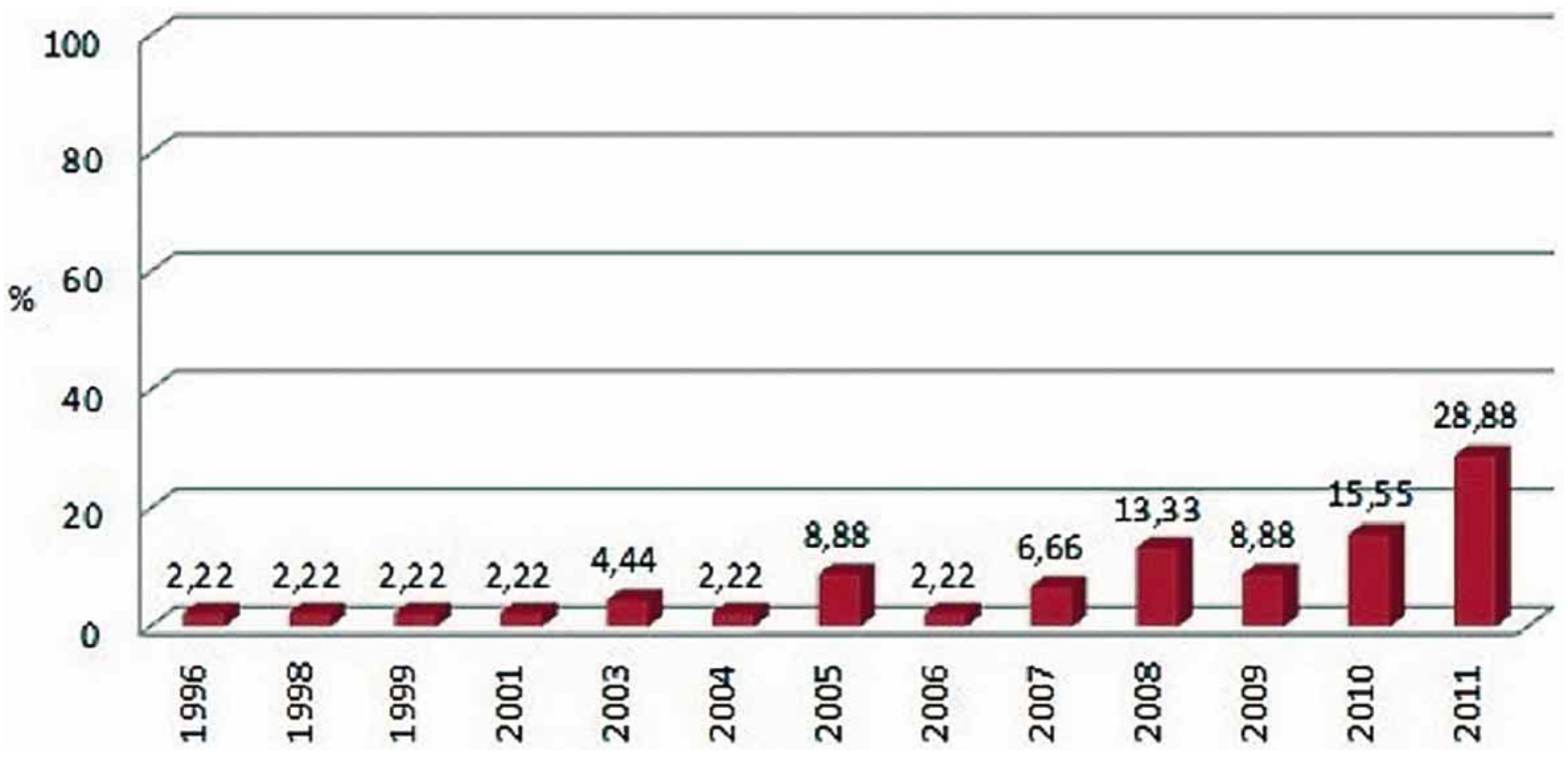

Fig. 2. Distribución del número de demandas presentadas por año, Ribeirão Preto, São Paulo, Brasil. 
TERADA, A. S. S. D.; DE ARAUJO, L. G.; FLORES, M. R. P. \& DA SILVA, R. H. A. Responsabilidad civil del cirujano-dentista: Análisis de las demandas presentadas en el municipio de Ribeirão Preto-SP, Brasil. Int. J. Odontostomat., 8(3):365-369, 2014.

das presentadas, se puede observar un aumento en los últimos años (Fig. 2). El Coeficiente de Experiencia Procesal en Ribeirão Preto en el año de 2011 fue de 5,92, según lo observado en la Tabla I. Las principales especialidades de Odontología involucradas en cuestiones procesales fueron en orden decreciente: prótesis dental $35,6 \%$; implante $26,6 \%$; endodoncia $15,5 \%$; ortodoncia $8,8 \%$; clínica general $6,6 \%$; endodoncia y prótesis dental $4,4 \%$; y cirugía $2,2 \%$.

Analizando las indemnizaciones solicitadas por los pacientes, se observa que los valores varían de $\mathrm{R} \$ 460,00$ (Reales Brasileños) (cerca de \$200,00 dólares) a $\mathrm{R} \$ 136.271,00$ (cerca de $\$ 59.300,00$ dólares), correspondiendo al valor mínimo y al valor máximo respectivamente, observando una media de $\mathrm{R} \$$
23.199,70 (aproximadamente $\$ 10.100,00$ dólares), una desviación estándar de 26.328,61 y error estándar de 4.015,07. La demanda de empresas de seguro de responsabilidad civil contratadas por cirujanos-dentistas fue observada en dos casos, representando un $4,4 \%$ de los procesos.

En el momento en que fueron investigados, fue posible observar que los procesos presentaban en diferentes etapas de juicio, en 33,3\% de los procesos había examen odontológico pericial. En relación a los procesos ya concluídos, 33,3\% ya habían sido juzgados, conforme Figura 3. Entre los casos resueltos a favor de los pacientes, se observó que la indemnización mínima pagada fue de $\mathrm{R} \$ 2.330,00$ y la máxima de $\mathrm{R} \$ 14.150,00$.

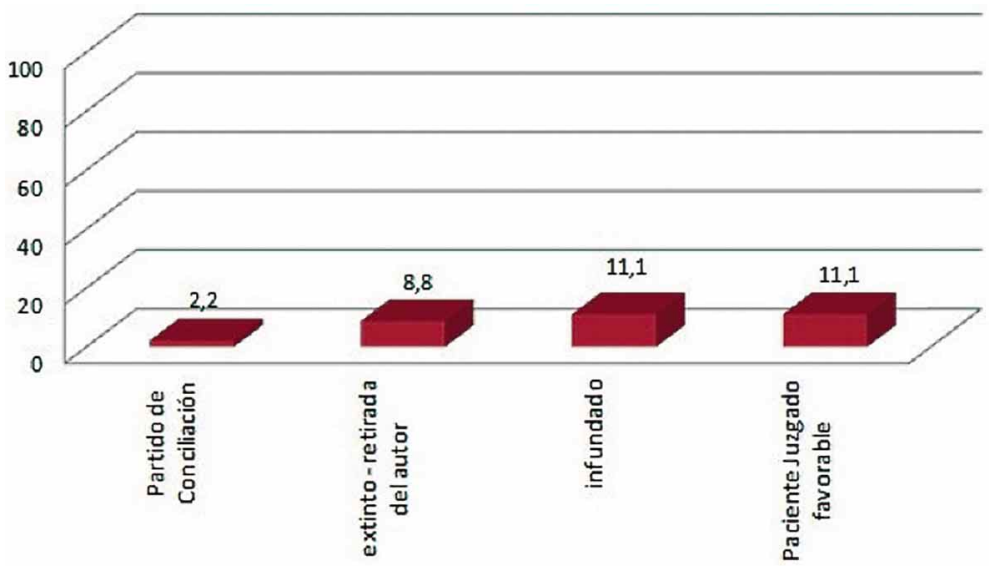

Fig. 3. Demandas de responsabilidad civil contra cirujanos-dentistas juzgadas, Ribeirão Preto, São Paulo, Brasil.

Tabla I. Coeficiente de Experiencia Procesal en 2011, Ribeirão Preto, São Paulo, Brasil.

\begin{tabular}{ccc}
$\begin{array}{c}\text { Número de demandas de } \\
\text { Responsabilidad Civil contra } \\
\text { Cirujanos-dentistas en 2011 }\end{array}$ & $\begin{array}{c}\text { Número de cirujanos-dentistas } \\
\text { y clíni cas registradas en el } \\
\text { municipio en 2011 }\end{array}$ & $\begin{array}{c}\text { Coeficiente de } \\
\text { Experiencia Procesal } \\
\text { en 2011 }\end{array}$ \\
\hline 13 & 2194 & 5,92 \\
\hline
\end{tabular}

\section{DISCUSIÓN}

El número de pacientes insatisfechos con el tratamiento recibido y que buscan de alguna forma, la reparación del daño sufrido, ha causado preocupación a la clase odontológica. El estudio de los procesos judiciales que involucran a los cirujanos-dentistas y a los establecimientos de asistencia odontológica en las diferentes esferas del derecho han sido objeto de estudio por algunos autores (de Paula et al., 2010; Santos et al.; Rosa et al., 2012; Wanderley e Lima et al., 2012). 
TERADA, A. S. S. D.; DE ARAUJO, L. G.; FLORES, M. R. P. \& DA SILVA, R. H. A. Responsabilidad civil del cirujano-dentista: Análisis de las demandas presentadas en el municipio de Ribeirão Preto-SP, Brasil. Int. J. Odontostomat., 8(3):365-369, 2014.

Por medio del presente estudio, fue posible trazar un panorama de la situación de las demandas relacionadas con responsabilidad civil del municipio de Ribeirão Preto, São Paulo, Brasil. En la Figura 2, es posible observar el creciente número de acciones interpuestas principalmente en los últimos cinco años. Tal hecho acompaña una realidad en el país. En el análisis de la jurisprudencia civil que comprende a estos profesionales en los tribunales de Brasil, fue posible observar la evolución del número de demandas a lo largo de los años en los tribunales brasileños (de Paula et al.).

El estudio que examinó las acciones civiles del período de 2000 a 2010 también reveló este aumento en el número de demandas a lo largo de los años. De un total de 201 sentencias analizadas, $31 \%$ eran de 2009 y $22 \%$ de 2010 (Walczewski et al., 2010).

Para observar si existe un determinado fenómeno en relación a la población expuesta, se indica el uso de coeficientes. El valor del CEP permite relacionar diferentes épocas y localidades, a través del uso de un artificio matemático. En el año 2006, al aplicar este coeficiente en Brasil, fue observado un valor igual a 2,23; cuando se analizan todos los procesos del Tribunal de Justicia, dividiéndolos por el número de profesionales registrados en el país (de Paula et al.). Un valor elevado de CEP $(5,92)$ fue encontrado en el municipio en el año 2011 en la localidad de Ribeirao Preto. Tal hecho puede ser explicado por el aumento significativo en el número de demandas en los últimos años, como es demostrado en este estudio.

Las áreas odontológicas más involucradas revelaron una similitud con los otros estudios realizados (de Paula et al.; Walczewski et al.; Wanderley e Lima et al.), en los cuales las especialidades de prótesis e implantología estaban entre las más demandadas. De la misma forma que es posible observar en este estudio, Ramos et al. (2000) al observar los procesos en el ámbito penal del estado de São Paulo, y Tanaka al analizar los reclamos contra cirujanos-dentistas en el Programa Estadual de Defensa del Consumidor (PROCON), verificaron que el área más demandada fue la de prótesis dental.

Una de las explicaciones al hecho de que estas áreas sean objeto de reclamaciones es por estar muy relacionadas con la expectativa estética de estos pacientes (Oliveira et al., 2010; Rosa et al.). Por ese motivo, los profesionales que trabajan en estas especialidades deben estar atentos para no generar altas expectativas en sus pacientes, prometiendo un resultado que podrá ser causal para la presentación de una demanda, si el tratamiento no finaliza como el paciente imaginaba.

Los valores de la indemnización solicitada fueron próximos a los observados por Costa-e-Silva \& Zimmermann (2006) en el estudio de los acuerdos en las páginas de los Tribunales de Justicia de las regiones Sur y Sudeste de Brasil, en relación a las acciones de responsabilidad civil contra cirujanos-dentistas del periodo 2005-2006, donde encontraron una variación de $R \$ 500,00$ a $R \$ 140.000,00$.

Estas similitudes encontradas, en relación a las áreas odontológicas más afectadas, al aumento progresivo del número de demandas, así como de los valores indemnizatorios requeridos, indican que las demandas judiciales del municipio de Ribeirão Preto representan muy bien la realidad de la jurisprudencia estadual y nacional encontrada.

Para auxiliar a los magistrados en la averiguación del daño y en la existencia del nexo causal, generalmente es solicitado el examen pericial del autor de la acción. Durante el trabajo pericial es responsabilidad del experto llevar las informaciones técnicas referentes a odontología al juez, por medio de los laudos periciales. La pericia es la búsqueda de pruebas que la justicia requiere para aclarar puntos que involucran el hecho (Silva). En este estudio, la presencia del examen pericial odontológico fue observada en un $33,3 \%$ de los casos. Este valor puede ser explicado por dos posibles causas: por la etapa en la que se encontraba el proceso y la cual podría no haber llegado a la fase de confirmación de la existencia del daño; o también por la posibilidad de que haya ocurrido conciliación de las partes en las fases iniciales del proceso, no llegando hasta la etapa del examen pericial.

Se puede concluir que el aumento en el número de demandas demostrado en el estudio evidencia un aumento de los litigios en la relación entre el profesional y el paciente. En este contexto, se torna importante el conocimiento del cirujano-dentista acerca de esta nueva realidad, para mejorar su práctica profesional y actual de forma más segura, evitando así procesos judiciales.

AGRADECIMIENTOS. A la Fundación Mapfre que financió la investigación. 
TERADA, A. S. S. D.; DE ARAUJO, L. G.; FLORES, M. R. P. \& DA SILVA, R. H. A. Civil liability of dental surgeons: legal proceedings analysis in the city of Ribeirao Preto, Sao Paulo State, Brazil. Int. J. Odontostomat., 8(3):359-364, 2014.

ABSTRACT: The objective of this study was to conduct a review of the legal proceedings involving liability lawsuits filed against dentists in Ribeirão Preto, São Paulo, Brazil. A search was carried out of professionals duly registered with the Regional Council of Dentistry of São Paulo seeking professionals and clinics involved in civil proceedings in Ribeirão Preto - São Paulo. The results noted a significant increase $(73.3 \%)$ in the number of lawsuits filed in the last five years, with the specialty in dental prostheses $(35.6 \%)$ being the most frequent. It can be concluded that it is important for professionals to have information in reference to the increasing number of lawsuits in professional/patient relationship and that prevention measures are necessary for a safer dental practice.

KEY WORDS: dental surgeons, legal dentistry, civil responsibility, proceedings.

\section{REFERENCIAS BIBLIOGRÁFICAS}

Costa e Silva, A. P. A. \& Zimmermann, R. D. Estudo dos acórdãos dos tribunais de justiça acerca das ações de responsabilidade civil contra cirurgiões-dentistas. Braz. Oral. Res., 20 Suppl.:286, 2006.

de Paula, F. J.; da Motta, M. V.; Bersácola, R. N. \& Muñoz, D. R. Panorama das ações de responsabilidade civil contra o odontólogo nos tribunais do Brasil. Rev. Paul. Odontol., 32(4):22-8, 2010.

Kfouri-Neto, M. Responsabilidade civil do médico. $4^{\text {a }}$ ed. São Paulo, Revista dos Tribunais, 2002. pp.152.

Nader, P. Introdução ao estudo do direito. $23^{\circ}$ ed. Rio de Janeiro, Forense, 2003. pp.418.

Oliveira, O. F.; Brand, T. M.; Benedicto, E. M.; Daruge, E. Jr. \& Paranhos, L. R. Documentação odontológica em implantodontia. In: Linden, A. S. S.; De Carli, J. P. \& Della Bona, A. (Eds.). Abordagens multidisciplinares na implantodontia. Porto Alegre, Editora RGO, 2010. Vol. 1. pp.16-20.

Pereira, C. M. S. Responsabilidade civil. $9^{\mathrm{a}}$ ed. Rio de Janeiro, Forense, 1998.

Ramos, D.; Gomes, E. M.; Frugoli, U. \& Cardozo, H. F. Processos de responsabilidade profissional contra o cirurgião-dentista em âmbito penal. Anais Forense, 2000. Disponible en: http:// www.ibemol.com.br/forense2000/093.asp

Ribeiro, A. R. M. D. Erros profissionais e seus aspectos jurídicos em odontologia legal. Rev. Bras. Odontol., 53(3):41-3, 1996.

Rosa, F. M.; Fernandes, M. M.; Daruge Júnior, E. \& Paranhos, L. R. Danos materiais e morais em processos envolvendo cirurgiõesdentistas no estado de São Paulo. Rev. Fac. Odontol. U. P. F., 17(1):26-30, 2012

Santos, F. S.; Scannavino, F. L. F.; Martins, A. T.; Barbosa, D. S.; Martins, D. A. \& Oliveira, R. M. D. Defesa do consumidor e a Odontologia: relato de caso. Cienc. Cult., 6(2):27-30, 2010.

Senado do República Federativa do Brasil. Constituição da República Federativa do Brasil. Artigo $5^{\circ}$, incisos V. Brasília (DF), Senado do República Federativa do Brasil, 1988.

Senado do República Federativa do Brasil. Lei n. 10.406 de 10 de janeiro de 2002: Institui Código Civil Brasileiro. Brasília (DF), Diário Oficial da União, Senado do República Federativa do Brasil, 2002.
Senado do República Federativa do Brasil. Lei n. 8078 de 11 de setembro de 1990: dispõe sobre a proteção do consumidos e dá outras providências. Brasília (DF), Diário Oficial da União, Senado do República Federativa do Brasil, 1990.

Serra, M. C. Responsabilidade profissional em odontologia: cuidados observados por cirurgiões dentistas com a documentação odontológica em consultórios particulares. Tese (Livre Docência). Araraquara, U. N. E. S. P., Faculdade de Odontologia, 2001.

Silva, R. H. A. Orientação profissional para o cirurgião-dentista: Ética e Legislação. São Paulo, Santos, 2010. pp.594.

Simonetti, F. A. A. Responsabilidade civil do cirurgião-dentista. Rev. Assoc. Paul. Cir. Dent., 53(6):449-51, 1999.

Tanaka, H. Verificação das reclamações contra cirurgiões dentistas no Procon de Presidente Prudente - SP. Araçatuba, U. N. E. S. P., Faculdade de Odontologia, 2002.

Walczewski, A. C. B.; de Oliveira, R. N. \& Kanto, E. A. Análise da jurisprudência brasileira frente a processos civis envolvendo a responsabilidade profissional do cirurgião-dentista. São Paulo, Anais XIII Congresso de Iniciação Científica da Universidade de Mogi das Cruzes, 2010. Disponible en: http://www.umc.br/_img/ diversos/pesquisa/pibic_pvic/XIII_congresso/projetos/ Ana\%20Claudia\%20Barzotto\%20Walczewski.pdf

Wanderley e Lima. R. B.; Moreira, V. G.; Cardoso, A. M. R.; Nunes, F. M. R.; Rabello, P. M. \& Santiago, B. M. Levantamento das Jurisprudências de Processos de Responsabilidade Civil Contra Cirurgiões-Dentistas nos Tribunais de Justiça Brasileiros. Rev. Bras. Cienc. Saúde, 16(1):49-58; 2012

Dirección para Correspondencia: Andrea Sayuri Silveira Dias Terada

Faculdade de Medicina de Ribeirão Preto - USP

Departamento de Patologia e Medicina Legal - CEMEL

Rua Tenente Catão Roxo, 2418

Monte Alegre - Ribeirão Preto/ SP

BRASIL

Email: andrea.terada@usp.br 